\title{
A Stability Analysis for Perturbed Nonlinear Iterative Methods
}

\author{
By Paul T. Boggs and J. E. Dennis, Jr.* \\ Dedicated to the memory of Kenneth $M$. Levenberg
}

\begin{abstract}
This paper applies the asymptotic stability theory for ordinary differential equations to Gavurin's continuous analogue of several well-known nonlinear iterative methods. In particular, a general theory is developed which extends the OrtegaRheinboldt concept of consistency to include the widely used finite-difference approximations to the gradient as well as the finite-difference approximations to the Jacobian in Newton's method. The theory is also shown to be applicable to the LevenbergMarquardt and finite-difference Levenberg-Marquardt methods.
\end{abstract}

1. Introduction. Many practical problems in applied science give rise to one of the following finite-dimensional problems.

(A) Given $f: R^{n} \rightarrow R^{1}$, find $x^{*} \in R^{n}$ at which $f$ achieves a minimum.

(B) Given $F: R^{n} \rightarrow R^{n}$, find $x^{*} \in R^{n}$ for which $F\left(x^{*}\right)=0$.

(C) Given $F: R^{n} \rightarrow R^{m}, m \geqslant n$, find $x^{*} \in R^{n}$ at which $\|F(x)\|_{2}$ achieves a minimum.

Usually, an acceptable approximation to $x *$ is sought by means of an iterative process of the form

$$
x_{k+1}=x_{k}-t_{k} G\left(x_{k}\right), \quad k=0,1, \ldots,
$$

where $x_{0}$ is a given initial approximation, $G$ is a vector valued function such that $G\left(x_{k}\right)$ indicates the direction to be taken by the iteration from $x_{k}$, and $t_{k}$ is a scalar which determines the magnitude of the step.

For example, in problem (A), $G\left(x_{k}\right)$ might be taken to be $\nabla f\left(x_{k}\right)$ and $t_{k}>0$ might be chosen to minimize $f\left(x_{k}-t G\left(x_{k}\right)\right)$ with respect to $t$ in some interval $(0, \bar{t}]$. This is, of course, the steepest descent method. Newton's method for problem (B) takes $G\left(x_{k}\right)=F^{\prime}\left(x_{k}\right)^{-1} F\left(x_{k}\right)$. In this case and in the one below, $t_{k}$ is initially chosen to prevent divergence and eventually taken to be unity in order to give the maximum rate of convergence. In problem (C) one might use the Levenberg-Marquardt method which takes

$$
G\left(x_{k}\right)=\left[\mu_{k} I+F^{\prime}\left(x_{k}\right)^{T} F^{\prime}\left(x_{k}\right)\right]^{-1} F^{\prime}\left(x_{k}\right)^{T} F\left(x_{k}\right) \text { where } \mu_{k} \geqslant 0 .
$$

(If $\mu_{k} \equiv 0$, then the Gauss-Newton method results.) Notice that in all these examples the point $x^{*}$ is a zero of $G$.

Received June 24, 1974; revised June 9, 1975.

AMS (MOS) subject classifications (1970). Primary 65H10; Secondary 65K05, 65005.

Key words and phrases. Nonlinear iterative methods, stability analysis, consistent approximations, steepest descent, Newton's method, nonlinear least squares methods.

* Research supported by National Science Foundation Grant number GJ-40903. 
These basic methods all have in common a need for first derivative information. Very often such information is either not available at all or else extremely expensive. In such cases it has long been standard procedure to use the basic algorithms with difference quotients in place of derivatives. Thus, if one needs

$$
\partial_{j} f(x)=\partial f(x) / \partial x_{j} \quad \text { for } f: R^{n} \rightarrow R^{1},
$$

one might use in its place

$$
\delta_{j} f(x, h)=\frac{f\left(x+h_{j} u_{j}\right)-f(x)}{h_{j}}, \quad h_{j} \neq 0, \quad j=1, \ldots, n,
$$

where $u_{j}=\left(\delta_{1 j}, \ldots, \delta_{n j}\right)^{T}$ and $\delta_{i j}$ is the Kronecker $\delta$-function. It is also possible to use a central difference quotient in place of (1.3), and we will remark on this at appropriate places in the sequel.

Theoretical and computational results surveyed in [6] indicate that if one chooses $h$ properly, then the finite-difference forms of the Newton and Levenberg-Marquardt algorithms can perform very well indeed.

In particular, it is shown that $h$ should be chosen as a suitable multiple of $\|F(x)\|$ or $\left\|F^{\prime}(x)^{T} F(x)\right\|$, respectively. Thus, the successive values of $h$ decrease and this in turn raises the real possibliity of cancellation errors in the finite precision computation of $\delta_{j} f(x, h)$ which would swamp the small truncation error, $\partial_{j} f(x)-\delta_{j} f(x, h)$, associated with $h$.

The use of a central difference only postpones the problem for one or two steps, and so it seems clear that any reasonable implementation of these ideas must admit the possibility that $h$ is eventually held fixed in the iteration.

In using Newton's method as described above, this does not affect convergence to $x^{*}$, but does, at least theoretically, slow to linear the rate of convergence. In using steepest descent however, $\nabla f$ will not in general have the same roots as its finite-difference approximation which implies that convergence to $x^{*}$ is no longer guaranteed. The purpose of this paper, therefore, is to compute a bound between $x^{*}$ and the limit of a sequence $\left\{x_{k}\right\}$ generated by using such an iteration. The bound obtained is a natural function of $h$ and the conditioning of the problem.

The nonlinear least squares problem is of special interest because it illustrates another way in which a parameter can enter the iteration. The Levenberg-Marquardt method [13] , [14], results from taking $G\left(x_{k}\right)=\left(\mu_{k} I+F^{\prime}\left(x_{k}\right)^{T} F^{\prime}\left(x_{k}\right)\right)^{-1} F^{\prime}\left(x_{k}\right)^{T} F\left(x_{k}\right)$, where $\mu_{k}$ is a nonnegative scalar. If $F^{\prime}\left(x_{k}\right)$ has less than full rank, then the implementation must either allow $\mu_{k}$ to eventually be held constant at some positive value or else switch to the more costly and less understood Ben-Israel iteration, $G(x)=F^{\prime}(x)^{+} F(x)$. (Here $A^{+}$denotes the Moore-Penrose inverse [19] of the rectangular matrix $A$.)

Our approach to these questions is somewhat unusual and so we outline it here. Following Gavurin [8], we define the continuous analogue to (1.1). This is the initial value problem

$$
x^{\prime}(s)=-G(x), \quad 0 \leqslant s<\infty, \quad x(0)=x_{0} .
$$


Notice that if Euler's method is applied to (1.4), then our original iteration (1.1) is recovered. The stability theory for ordinary differential equations identifies conditions on $G$ and $x_{0}$ that imply the existence of a solution curve $x(s)$ such that $\lim _{s \rightarrow \infty} x(s)=$ $x^{*}$. This provides quantitative information on the directions to be used. Although the connection is well known it usually fails to say anything new about the convergence of the iterations.

On the other hand, we are not so much interested in iterations of the form (1.1) as we are in those of the form

$$
x_{k+1}=x_{k}-t_{k} \widetilde{G}\left(x_{k}, p\right)
$$

where $p$ is some parameter vector. The corresponding continuous analogue is

$$
x^{\prime}(s)=-\widetilde{G}(x, p), \quad 0 \leqslant s<\infty, \quad x(0)=x_{0} .
$$

We write (1.6) as $x^{\prime}(s)=-G(x)+[G(x)-\widetilde{G}(x, p)]$ and view (1.6) as a perturbation, controlled by $p$, to (1.4). In this case, the stability theory again provides qualitative information on solutions $x_{p}(s)$ to (1.6). Moreover, it also provides a bound on $\varlimsup_{s \rightarrow \infty}\left\|x_{p}(s)-x *\right\|$ in terms of $p, G$ and $x_{0}$. The application of Euler's method to (1.6) now yields a similar bound on $\lim _{k \rightarrow \infty}\left\|x_{k}-x *\right\|$ where $x_{k}$ is a solution to (1.5).

Clearly, some conditions on $\|G(x)-\widetilde{G}(x, p)\|$ are necessary for such a result. In Section 2, we generalize the Ortega-Rheinboldt [17] idea of a consistent Jacobian approximation to provide a sufficient condition; and we also show that the specific instances of (1.5) mentioned earlier satisfy our condition. Sections 3 and 4 are devoted to the minimal amount of stability theory for (1.6) and (1.5) necessary for the results on specific methods which we present in Section 5 .

It is perhaps worth noting at this point that other authors, including Meyer [15] and Bosarge [3] , have used the continuous analogue, numerical integration connection; but they work with a finite range of the independent variable. The advantages of our approach are set forth in Boggs [1] and provide motivation for our choice of an infinite range.

An interesting different approach to the analysis of iterative methods by differential equation techniques was given by Hurt [12]. In his paper, discrete analogues of Liapunov functions are defined and used to prove certain stability results. These results are then used, for example, to obtain regions of convergence for Newton's method and to analyze the effects of round-off error. However, the resulting bounds depend on the Liapunov functions used; and thus, sharp bounds are quite difficult to obtain. Also, for some iterative methods, finding any Liapunov function may require considerable ingenuity. Nevertheless, this approach does seem valuable and has been used by Boggs [2] to analyze certain algorithms in the presence of singularities. (See also Ortega [16] for a bibliography and an exposition of the basic results.)

2. Consistent Approximations. In order to present a unified theory for the various multidimensional secant methods as well as the modification of Newton's method in which the Jacobian matrix is replaced by the corresponding matrix of difference quotients, Ortega and Rheinboldt [17, p. 355] employ a very elegant formalism called a 
"consistent approximation" to the Jacobian. A generalization of this concept to other than Jacobians will be useful to us.

We use $\bar{P}$ to denote the closure of a set $P$.

Definition 2.1. Let $D \subset R^{n}, P \subset R^{q}$ with $0 \in \bar{P}$. Let $G$ be a mapping of $D$ into $R^{m}$. A function $\widetilde{G}$ of the vector variables $(x, p)$ defined on $D \times P$ is a consistent approximation rule for $G$ on $D$ if and only if

$$
\|G(x)-\widetilde{G}(x, p)\| \rightarrow 0 \text { as }\|p\| \rightarrow 0,
$$

uniformly on every compact subset of $D$. In addition, if there are positive constants $\alpha, \beta$ and $\epsilon$ such that for every $x \in D$ and $\|p\|<\epsilon$,

$$
\|G(x)-\widetilde{G}(x, p)\| \leqslant \beta\|p\|^{\alpha},
$$

then $\widetilde{G}$ is a strongly consistent approximation rule of order $\alpha$ for $G$ on $D$.

Clearly, the property of consistency and the value of $\alpha$ are norm independent while $\beta$ is not.

We now proceed to show that the iterations mentioned in the introduction are connected by this concept. In the lemma below we will assume that the functional $f$, defined from an open convex set $D \subset R^{n}$ into the real numbers, is continuously differentiable on $D$. If $x \in D$, then (1.2) is defined; but we must restrict $x$ and $h$ if (1.3) is to be defined. Choose $D_{0} \subset D$ such that $\bar{D}_{0} \subset D$ and choose $\epsilon>0$ such that $D \supset N\left(D_{0}, \epsilon\right) \equiv\left\{x \in R^{n}:\|x-y\|<\epsilon\right.$ for some $\left.y \in D_{0}\right\}$. Now for $(x, h) \in D_{0} \times$ $(0, \epsilon]^{n}, \delta_{j} f(x, h)$ is well defined by $(1.3)$ for $1 \leqslant j \leqslant n$.

It is sometimes the case that the analyst might choose to compute some partial derivatives of $f$ and approximate others. Hence, we define our approximation to the gradient as follows. For $(x, h) \in D_{0} \times[0, \epsilon]^{n}$ take $\Delta f(x, h)$ to be a column vector where, for $i=1, \ldots, n$,

$$
\Delta f(x, h)_{i}= \begin{cases}\delta_{i} f(x, h), & h_{i} \neq 0, \\ \partial_{i} f(x), & h_{i}=0 .\end{cases}
$$

It is obvious that we intend $\Delta f(x, h)$ as an approximation to the derivative of $f$ at $x$. Since this derivative is, strictly speaking, a linear functional and so more naturally represented as a row vector, while $\Delta f(x, h)$ is a column vector, we invoke the Riesz Representation Theorem once here in order to avoid frequent use of superscript $T$ and make the notational convention that $\nabla f(x)$ is the column vector which represents $f^{\prime}(x)$ (cf. [9, p. 116] or Tapia [20]). There is no need to be precise when dealing with $f^{\prime \prime}(x)$.

We omit the proof of the following lemma since it is so similar to the proof given in $[17$, p. 359].

LEMMA 2.1. Let $f$ satisfy the conditions given above, then $\Delta f$ is a consistent approximation rule for $\nabla f$ on $D_{0}$. Furthermore, if for some $K, \alpha>0$ and every $x, y \in D$,

$$
\|\nabla f(x)-\nabla f(y)\| \leqslant K\|x-y\|^{\alpha}
$$


then $\Delta f$ is a strongly consistent approximation rule of order $\alpha$ for $\nabla f$ on $D_{0}$.

The following lemma proved in [7], is also just a restatement of a well-known result. We establish our notation before the statement. Let $F=\left(f_{1}, \ldots, f_{m}\right)^{T}$ be a continuously differentiable mapping from $D$ into $R^{m}$ where $D$ is an open convex subset of $R^{n}$. Choose $D_{0} \subset D$ and $\epsilon>0$ as before and define an approximation to the Jacobian matrix for $F$ as follows. For $(x, h) \in D_{0} \times[0, \epsilon]$ set

$$
\Delta F(x, h)=\left[\begin{array}{c}
\Delta f_{1}(x, h)^{T} \\
\vdots \\
\Delta f_{i}(x, h)^{T} \\
\vdots \\
\Delta f_{m}(x, h)^{T}
\end{array}\right] .
$$

LEMMA 2.2. Let $F$ satisfy the conditions given above, then $\Delta F$ is a consistent approximation rule for $F^{\prime}$ on $D_{0}$. Furthermore, if for some $K, \alpha>0$, and every $x, y \in D$,

$$
\left\|F^{\prime}(x)-F^{\prime}(y)\right\| \leqslant K\|x-y\|^{\alpha},
$$

then $\Delta F$ is a strongly consistent approximation rule of order $\alpha$ for $F^{\prime}$ on $D_{0}$.

Let us now consider a quite different example.

THEOREM 2.3. Let $F$ map a bounded open convex subset $D$ of $R^{n}$ into $R^{m}$ where $m \geqslant n$. In addition, assume that $F$ is continuously differentiable on $D$ and that the rank of $F^{\prime}(x)$ is the same for every $x \in D$. For $\mu>0$ and $x \in D$, define the Levenberg approximation to $F^{\prime}(x)^{+}$by $L(x, \mu)=\left(\mu I+F^{\prime}(x)^{T} F^{\prime}(x)\right)^{-1} F^{\prime}(x)^{T}$. Then the Levenberg approximation is strongly consistent of order 1 with respect to the parameter $\mu$.

Proof. $F^{\prime}(x)^{+}$is a continuous function of $x$ on $D$ since $F$ is continuously differentiable and $F^{\prime}(x)$ has constant rank on $D$ (cf. [10, Theorem 4.3]). Let $F^{\prime}(x)=$ $B(x) D(x) C(x)^{T}$ be a singular value decomposition of $F^{\prime}(x)$ with $B(x)$ and $C(x)$ unitary square matrices of order $m \times m$ and $n \times n$, respectively. Then $F^{\prime}(x)^{+}=C(x) D(x)^{+} B(x)^{T}$. Therfore, we drop the argument $x$ and remembering not to confuse $D(x)$ with the domain $D$ we write

$$
\begin{aligned}
E(x, \mu) & =F^{+}-\left(\mu I+F^{\prime T} F^{\prime}\right)^{-1} F^{\prime T}=C D^{+} B^{T}-\left(\mu I+C D^{T} D C^{T}\right)^{-1} C D^{T} B^{T} \\
& =C\left[D^{+}-\left(\mu I+D^{T} D\right)^{-1} D^{T}\right] B^{T} .
\end{aligned}
$$

Now let $D(x)$ equal $\left(\begin{array}{cc}\Lambda(x) & 0 \\ 0 & 0\end{array}\right)$ where $\Lambda(x)$ is the $r \times r$ diagonal matrix of positive singular values $\lambda_{1}(x), \ldots, \lambda_{r}(x)$ of $F^{\prime}(x)$. Thus,

$$
E(x, \mu)=C\left(\begin{array}{cc}
\Lambda^{-1}-\left(\mu I+\Lambda^{2}\right)^{-1} \Lambda & 0 \\
0 & 0
\end{array}\right) B^{T} .
$$

In order to bound $E(x, \mu)$, we first note that since the diagonal elements of $D(x)$ are the nonnegative square roots of the eigenvalues of $F^{\prime}(x)^{T} F^{\prime}(x)$, there is a neighborhood $N(x)$ about $x$ such that for $y \in N(x), \lambda_{i}(y)>\lambda_{i}(x) / 2$ for $1 \leqslant i \leqslant r$. (See [18, p. 282].) Choose a finite subcover $N\left(x_{1}\right), N\left(x_{2}\right), \ldots, N\left(x_{q}\right)$ of the compact set $\bar{D}$. Clearly then, for $x \in D$, 


$$
\lambda_{i}(x)>\min _{1 \leqslant j \leqslant q} \min _{1 \leqslant k \leqslant r} \lambda_{k}\left(x_{j}\right) / 2 \equiv \bar{\alpha} .
$$

We now return to the task of bounding $E(x, \mu)$. Since $\|C(x)\|_{2}=\|B(x)\|_{2}=1$, we need only bound $\left\|\Lambda^{-1}-\left(\mu I+\Lambda^{2}\right)^{-1} \Lambda\right\|$. But this is a nonnegative diagonal matrix so its $l_{2}$ norm is its maximum diagonal element,

$$
\max _{1 \leqslant i \leqslant r} \frac{1}{\lambda_{i}}-\frac{\lambda_{i}}{\mu+\lambda_{i}^{2}}=\mu \cdot \max _{1 \leqslant i \leqslant r} \frac{1}{\lambda_{i}\left(\mu+\lambda_{i}^{2}\right)} \leqslant \mu / \bar{\alpha}^{3},
$$

which completes the proof.

In proving convergence of the Ben-Israel iteration, we will need an estimate of $\left\|L(x, \mu) F(x)-F^{\prime}(x)^{+} F(x)\right\|$ which we obtain next.

Corollary 2.4. Assume the conditions of Theorem 2.3. Assume further that there is a point $x^{*} \in D$ such that $F^{\prime}\left(x^{*}\right)^{+} F\left(x^{*}\right)=0$ and

$$
\left\|F^{\prime}(x)^{+} F(x)-F^{\prime}\left(x^{*}\right)^{+} F\left(x^{*}\right)\right\| \leqslant K\left\|x-x^{*}\right\|^{\beta},
$$

for all $x \in D$. Then, for $\mu>0$,

$$
\left\|L(x, \mu) F(x)-F^{\prime}(x)^{+} F(x)\right\| \leqslant\left(\mu / \bar{\alpha}^{3}\right) \cdot K\left\|x-x^{*}\right\|^{\beta},
$$

for any $\bar{\alpha}>0$, which is a uniform lower bound on the nonzero singular values of $F^{\prime}(x), x \in D$.

Proof. Clearly, $L(x, \mu) F(x)=F^{\prime}(x)^{+} F(x)-E(x, \mu) F(x)$. Then, from (2.7) and the fact that $D^{T}(x) D(x) D^{+}(x)=D^{T}(x)$, we again drop the subscripts and write

$$
\begin{aligned}
E(x, \mu) F(x) & =C\left[D^{+}-\left(\mu I+D^{T} D\right)^{-1} D^{T}\right] B^{T} F=C\left[I-\left(\mu I+D^{T} D\right)^{-1} D^{T} D\right] D^{+} B^{T} F \\
& =C\left[I-\left(\mu I+D^{T} D\right)^{-1} D^{T} D\right] C^{T}\left[F^{+} F-F^{\prime}\left(x^{*}\right)^{+} F\left(x^{*}\right)\right] .
\end{aligned}
$$

Therefore,

$$
\begin{aligned}
\|E(x, \mu) F(x)\| & \leqslant\left\|I-\left(\mu I+D^{T} D\right)^{-1} D^{T} D\right\| \cdot K\left\|x-x^{*}\right\|^{\beta} \\
& \leqslant\left(\mu / \bar{\alpha}^{3}\right) K\left\|x-x^{*}\right\|^{\beta}
\end{aligned}
$$

which completes the proof.

In [4], Brown and Dennis gave theoretical and computational justification for the use of finite differences in the Levenberg and Gauss-Newton methods. We will prove the consistency of the finite-difference Levenberg approximation to $F^{\prime}(x)^{+}$. For $\mu>0,\|h\|<\epsilon, x \in D_{0}$ set

$$
\widetilde{L}(x,(\mu, h))=\left(\mu I+\Delta F(x, h)^{T} \Delta F(x, h)\right)^{-1} \Delta F(x, h) ;
$$

but we must restrict the parameter vector $p=(\mu, h)$ somewhat. Notice that if $\mu$ goes to zero faster than $h$, then there is no guarantee that $\Delta F(x, h)$ has the same rank as $F^{\prime}(x)$, and so, it is not clear that $\Delta F(x, h)^{+} \rightarrow F^{\prime}(x)^{+}$as $\|h\| \rightarrow 0$. Thus, we define the parameter set $P=\left\{p=(\mu, h) \in(0, \infty) \times N(0, \epsilon)\right.$; and if $\left\{p_{k}\right\} \rightarrow 0$ as $k \rightarrow \infty$, then $\left\|h_{k}\right\|^{\alpha} / \mu_{k}^{2} \rightarrow 0$ as $\left.k \rightarrow \infty\right\}$. Here $P \subset R^{n+1}$ and $\alpha$ is from (2.6).

THEOREM 2.5. Let $F$ satisfy the hypothesis of Theorem 2.3 as well as the Lipschitz condition (2.6). Then $\widetilde{L}$ as defined above on $D_{0} \times P$ is a consistent approximation to $F^{\prime}(x)^{+}$on $D_{0}$. 
Proof. Set $\widetilde{E}(x, p)=\widetilde{L}(x, p)-F^{\prime}(x)^{+}$for $x \in D_{0},(\mu, h)=p \in P$. If we add and subtract $L(x, \mu)$, we need only show that $\|\widetilde{L}(x, p)-L(x, \mu)\| \rightarrow 0$ as $\|p\| \rightarrow 0$ uniformly on compact subsets of $D_{0}$, since, by Theorem $2.3,\left\|L(x, \mu)-F^{\prime}(x)^{+}\right\| \rightarrow 0$ as $\|p\| \rightarrow 0$ independent of $x \in D_{0}$. Now for $p=(\mu, h) \in P$,

$$
\begin{aligned}
\widetilde{L}(x, p)-L(x, \mu)= & \widetilde{L}(x, p)-\left(\mu I+\Delta F(x, h)^{T} \Delta F(x, h)\right)^{-1} F^{\prime}(x)^{T} \\
& +\left(\mu I+\Delta F(x, h)^{T} \Delta F(x, h)\right)^{-1} F^{\prime}(x)^{T}-L(x, \mu) .
\end{aligned}
$$

The first two terms on the right reduce to

$$
\left(\mu I+\Delta F(x, h)^{T} \Delta F(x, h)\right)^{-1}\left(F^{\prime}(x)^{T}-\Delta F(x, h)^{T}\right),
$$

which is bounded in norm by $C K\|h\|^{\alpha} / \mu$ for some $C$. The second two terms reduce to

$$
\left[\left(\mu I+\Delta F(x, h)^{T} \Delta F(x, h)\right)^{-1}-\left(\mu I+F^{\prime}(x)^{T} F^{\prime}(x)\right)^{-1}\right] F^{\prime}(x)^{T} .
$$

Since $F^{\prime}(x)$ is uniformly bounded on compact subsets of $D_{0}$, we need only consider the difference of the inverses. Each inverse is bounded in norm by $1 / \mu$; and so, the difference is bounded by

$$
\left\|F^{\prime}(x)^{T} F^{\prime}(x) \pm F^{\prime}(x)^{T} \Delta F(x, h)-\Delta F(x, h)^{T} \Delta F(x, h)\right\| / \mu^{2}
$$

which is $O\left(\|h\|^{\alpha} / \mu^{2}\right)$ on any compact subset of $D_{0}$. If $p \rightarrow 0$, then because of the definition of $P,\|h\|^{\alpha} / \mu^{2} \rightarrow 0$; and the proof is complete.

The situation is greatly simplified if $F^{\prime}(x)$ has full rank for every $x \in D_{0}$. There is no longer any need for the restriction of $P$ or for condition (2.6).

TheOREM 2.6. Let $F$ satisfy the hypothesis of Theorem 2.5, and assume in addition that the rank of $F^{\prime}(x)$ is $n$ for every $x \in D_{0}$. Let $P_{0}=\{p=(\mu, h): \mu>0, h \in$ $E^{n}$, and $\left.\|h\|<\epsilon\right\}$. Then $\widetilde{L}$ defined on $D_{0} \times P_{0}$ is a consistent approximation to $F^{\prime}(x)^{+}=\left[F^{\prime}(x)^{T} F^{\prime}(x)\right]^{-1} F^{\prime}(x)^{T}$.

Furthermore, if $D_{0}$ is compact then there is some $\widetilde{\epsilon}$ such that if $P_{1}=\{p=(\mu, h)$ : $\mu \geqslant 0, h \in E^{n}$ and $\left.\|h\|<\widetilde{\epsilon}\right\}$ then $\widetilde{L}$ defined on $D_{0} \times P_{1}$ is a strongly consistent approximation of order $\alpha$ to $F^{\prime}(x)^{+}$on $D_{0}$. In particular, the finite-difference GaussNewton method is a strongly consistent, order $\alpha$ approximation to the Gauss-Newton method in this case.

Proof. Let $D_{1}$ be a compact subset of $D_{0}$. Since $F$ is continuously differentiable and $F^{\prime}(x)$ is of full rank on $D_{1}$, the smallest eigenvalue of $F^{\prime}(x)^{T} F^{\prime}(x), \lambda(x)$ is a positive continuous function of $x$ on the compact set $D_{1}$. Hence, there is some positive $\lambda \leqslant \lambda(x)$ for every $x \in D_{1}$. From the definition of $\Delta F(x, h)$ and the hypotheses on $F$, for some constant $C,\left\|\Delta F(x, h)^{T} \Delta F(x, h)-F^{\prime}(x)^{T} F^{\prime}(x)\right\| \leqslant C\|h\|^{\alpha}$ on $D_{1}$. There is, hence, an $\widetilde{\epsilon}$ such that for any $x \in D_{1}$, and $\|h\| \leqslant \widetilde{\epsilon},\left(\Delta F(x, h)^{T} \Delta F(x, h)\right)^{-1}$ exists and is bounded in norm by $2 / \lambda$, i.e., the smallest eigenvalue of $\Delta F(x, h)^{T} \Delta F(x, h)$ is bounded below by $\lambda / 2$ for every $(x, h) \in D_{1} \times[0, \widetilde{\epsilon}]^{n}$. This is a standard Banach lemma argument and need not be detailed here.

We point out here that the reason $\mu=0$ is excluded from $P_{0}$ is that $\Delta F(x, h)$ does not necessarily have full rank for $\|h\|<\epsilon$. However, if $D_{0}$ is compact then $D_{1}$ in the above paragraph could be taken equal to $D_{0}$; and for $\|h\|<\widetilde{\epsilon}, \Delta F(x, h)$ has 
full rank so $\widetilde{L}(x,(0, h))$ is defined. Essentially, this is the only difference between the two parts of the proof.

From this point on, we argue as in the proof of Theorem 2.5 that we need only bound either side of (2.8), in this case, by $C\|h\|^{\alpha}$, to complete the proof. Break up the right side of (2.8) and note that for $\|p\|$ small enough, and some $\bar{C}$,

$$
\begin{aligned}
\|\left(\mu I+\Delta F(x, h)^{T} \Delta F(x, h)\right)^{-1}\left(F^{\prime}(x)^{T}-\right. & \left.\Delta F(x, h)^{T}\right) \| \\
& \leqslant \bar{C} K\|h\|^{\alpha} /(\mu+\lambda / 2)<2 \bar{C} K\|h\|^{\alpha} .
\end{aligned}
$$

Similarly,

$$
\left[\left(\mu I+\Delta F(x, h)^{T} \Delta F(x, h)\right)^{-1}-\left(\mu I+F^{\prime}(x)^{T} F^{\prime}(x)\right)^{-1}\right] F^{\prime}(x)^{T},
$$

is bounded in norm on $D_{1}$ for $\|p\|$ small enough by a constant times $\|h\|^{\alpha} /\{(\mu+\lambda / 2)(\mu+\lambda)\}$, and so is $O\left(\|h\|^{\alpha}\right)$, and the proof is complete.

3. Stability Analysis for the Continuous Analogue. This section is devoted to a development of asymptotic results for the continuous analogue initial value problems (1.4) and (1.6). Our technique will be to first view (1.6) as a perturbation of the continuous analogue initial value problem (1.4), which is in turn treated as a perturbation of an easily analyzed affine problem. Thus, we write (1.6) as

$$
x^{\prime}(s)=-A(x)+[A(x)-G(x)]+[G(x)-\widetilde{G}(x, p)] \quad x(0)=x_{0}, \quad 0 \leqslant s<\infty,
$$

where $A(x) \equiv A x-b$ is an affine transformation.

Before proceeding with the analysis, let us illustrate (3.1) with steepest descent. The discussion in Section 1 leads us to want an asymptotic analysis of

$$
\begin{aligned}
x^{\prime}(s) & =-\Delta f(x, h), \quad x(0)=x_{0}, \quad 0 \leqslant s<\infty, \\
& =-\nabla f(x)+[\nabla f(x)-\Delta f(x, h)] \\
& =-f^{\prime \prime}\left(x^{*}\right)\left(x-x^{*}\right)+\left[f^{\prime \prime}\left(x^{*}\right)\left(x-x^{*}\right)-\nabla f(x)\right]+[\nabla f(x)-\Delta f(x, h)] .
\end{aligned}
$$

Now if the Hessian at $x^{*}, f^{\prime \prime}\left(x^{*}\right)=\left(\partial_{i j} f\left(x^{*}\right)\right)$ is symmetric and positive definite, then it is well known (cf. [5, Chapter 3]) that the affine equation $x^{\prime}(s)=-f^{\prime \prime}\left(x^{*}\right) x+f^{\prime \prime}\left(x^{*}\right) x^{*}$ is solved by $x(s)-x^{*}=\left(x_{0}-x^{*}\right) e^{-f^{\prime \prime}\left(x^{*}\right) s}$, which (we will see) has the curve $\phi(s) \equiv x^{*}$ as an asymptote. If $x^{*}$ is a local minimizer for $f$, then $\nabla f\left(x^{*}\right)=0$; and so, the second group of terms can be written as the negative of a Taylor series remainder $[\nabla f(x)-$ $\left.\nabla f\left(x^{*}\right)-f^{\prime \prime}\left(x^{*}\right)\left(x-x^{*}\right)\right]$. Thus, in a neighborhood of $x^{*}$, the continuous analogue of steepest descent, $x^{\prime}(s)=-\nabla f(x)$, is a perturbation of the affine problem. If we assume $f^{\prime \prime}\left(x^{*}\right)$ is the Fréchet derivative of $\nabla f$ at $x^{*}$, then for any $\epsilon>0$ there is a $\delta>0$ such that this perturbation, associated with the idealized iteration, is bounded in norm by $\epsilon\left\|x-x^{*}\right\|$ for $\left\|x-x^{*}\right\|<\delta$. An asymptotic analysis for this case of the continuous analogue (1.4) can be accomplished by standard means, and its asymptotic behavior is essentially unchanged.

We have to extend the standard results in order to incorporate the third term into the stability theory for initial value problems. This term has the continuous analogue effect of using an approximation to the idealized iteration. Our extended theory cannot 
really be expected to imply the same sort of asymptotic convergence as before, since $\nabla f(x)-\Delta f(x, h)$ depends on $h$ instead of $x-x^{*}$.

The concept of consistency developed in Section 2 will allow us to draw some interesting conclusions about the behavior of solutions to (1.6) as $s$ becomes large. In the case of (1.4) under the usual hypotheses, we expect any solution $x(s)$ to have the property that for any $\epsilon$ there is an $S$ such that $\left\|x(s)-x^{*}\right\|<\epsilon$ for $s>S$. For (1.6) this property holds generally only for $\epsilon$ greater than some lower bound which depends on $\|p\|$. For steepest descent, we will conclude roughly that

$$
\varlimsup_{s \rightarrow \infty}\left\|x(s)-x^{*}\right\| \leqslant O\left(\|h\|^{\alpha} k_{2}\left(f^{\prime \prime}\left(x^{*}\right)\right)\right),
$$

where $k_{2}(\circ)$ is the $l_{2}$ condition number of the matrix argument.

We state the following lemma because the results will be useful; but we omit the proof because it is not central to this paper and it can be found elsewhere [5].

Lemma 3.1. Let $A$ be a real $n \times n$ matrix and let $A=C \Lambda C^{-1}$ be its Jordan canonical form. Assume that the real part of $\lambda$ is positive for $\lambda$ chosen to be the eigenvalue of $A$ with minimal real part. For any real $t$, define

$$
e^{-A t}=I+\sum_{k=1}^{\infty}(-1)^{k} t^{k} A^{k} / k ! .
$$

Then, there exist positive constants $v, \sigma$, such that $\left\|e^{-A t}\right\| \leqslant v e^{-\sigma t}$. The values of $\nu$ and $\sigma$ depend on $A$ and the norm as follows.

(i) If $A$ is symmetric, then $\sigma=\lambda$; and if the $l_{2}$ norm is used, $\nu=1$.

(ii) If the largest Jordan block corresponding to $\lambda$ is $1 \times 1$, then $\sigma$ is the real part of $\lambda$; and if the $l_{2}$ norm is used, $\nu=k_{2}(C)$.

(iii) If the largest Jordan block corresponding to $\lambda$ is not $1 \times 1$, then $\nu=k_{2}(C) / \sigma$ in the case of the $l_{2}$ norm; but in any case, $\sigma$ is one half the real part of $\lambda$.

Now we are ready to give the stability result we require. It is a modification of a well-known theorem which can be found in [5].

Theorem 3.2. Let $r \in(0,1)$ and let $D$ be an open convex neighborhood in $R^{n}$ of $x^{*}$, a zero of $G: D \rightarrow R^{n}$. Assume that the Fréchet derivative $G^{\prime}\left(x^{*}\right)=A$ exists, that its eigenvalues all have positive real parts; and let the constants $\nu$ and $\sigma$ be defined by Lemma 3.1. Finally, let $\widetilde{G}$ be a consistent approximation rule for $G$ on $D$. Then, there is an $\epsilon>0$ and a neighborhood $D_{r}$ of $x^{*}$ such that any solution to

$$
x^{\prime}(s)=-\widetilde{G}(x, p), \quad x(0)=x_{0} \in D_{r}, \quad\|p\|<\xi, \quad 0 \leqslant s,
$$

satisfies

$$
\varlimsup_{s \rightarrow \infty}\|x(s)-x *\| \leqslant\left[\sup _{x \in D}\|G(x)-\widetilde{G}(x, p)\|\right] \nu / \sigma(1-r) .
$$

Proof. From [5, p. 328], every solution of problem (3.1) satisfies

$$
\begin{aligned}
x(s)-x^{*}= & e^{-A s}\left(x(0)-x^{*}\right)+\int_{0}^{s} e^{-A(s-u)}[A(x)-G(x)] d u \\
& +\int_{0}^{s} e^{-A(s-u)}[G(x)-\widetilde{G}(x, p)] d u .
\end{aligned}
$$

Define $q(p)=\sup _{x \in D}\|G(x)-\widetilde{G}(x, p)\|$ and let $\epsilon<\sigma r / \nu$. Choose $\delta>0$ so that 
$\|A(x)-G(x)\|=\left\|A(x)-G(x)+G\left(x^{*}\right)\right\|<\epsilon\left\|x-x^{*}\right\|$ for $\left\|x-x^{*}\right\|<\delta$. By Lemma 3.1 we have

$$
\begin{aligned}
\|x(s)-x *\| \leqslant & \nu e^{-\sigma s}\left\|x_{0}-x^{*}\right\|+\nu \epsilon \int_{0}^{s} e^{-\sigma(s-u)}\|x(u)-x *\| d u \\
& +\nu \int_{0}^{s} e^{-\sigma(s-u)} q(p) d u .
\end{aligned}
$$

Since $\sigma>0 \leqslant s, e^{-\sigma s} \leqslant 1$ so for any $s \geqslant 0$,

$$
\left\|x(s)-x^{*}\right\| \leqslant \nu\left\|x_{0}-x^{*}\right\|+\nu \epsilon\left[\max _{0 \leqslant u \leqslant s}\left\|x(u)-x^{*}\right\|+\nu q(p)\right] / \sigma,
$$

which resolves to

$$
\max _{0 \leqslant u \leqslant s}\|x(u)-x *\| \leqslant \frac{\nu \sigma\left\|x_{0}-x^{*}\right\|}{\sigma-\nu \epsilon}+\frac{\nu q(p)}{\sigma-\nu \epsilon} \leqslant \frac{\left[\sigma \nu\left\|x_{0}-x^{*}\right\|+\nu q(p)\right]}{(1-r) \sigma} .
$$

Now choose $\xi$ and $D_{r}$ so that the right-hand side of (3.4) is bounded above by $\delta$.

It remains for us to bound $\gamma \equiv \varlimsup_{s \rightarrow \infty}\|x(s)-x *\|$. Choose a sequence $\left\{s_{j}\right\} \rightarrow \infty$ such that $\left\|x\left(s_{j}\right)-x *\right\| \rightarrow_{s \rightarrow \infty} \gamma$. From inequality (3.3) we obtain

$$
\begin{aligned}
\left\|x\left(s_{j}\right)-x^{*}\right\| \leqslant & \left\|x_{0}-x^{*}\right\| \nu e^{-\sigma s}+\nu \epsilon \int_{0}^{s_{j} / 2} e^{-\sigma\left(s_{j}-u\right)}\left\|x(u)-x^{*}\right\| d u \\
& +\nu \epsilon \int_{s_{j} / 2}^{s_{j}} e^{-\sigma\left(s_{j}-u\right)}\left\|x(u)-x^{*}\right\| d u \\
& +\nu \int_{0}^{s_{j}} e^{-\sigma\left(s_{j}-u\right)} q(p) d u .
\end{aligned}
$$

Let $\eta$ be arbitrary in the interval $(0, \gamma)$. Then there is an integer $j_{\eta}$ such that for every $j \geqslant j_{\eta}, \gamma-\eta<\left\|x\left(s_{j}\right)-x *\right\|<\gamma+\eta$. Thus for $j>j_{\eta}$, (3.5) implies

$$
\begin{aligned}
\gamma-\eta< & \left\|x_{0}-x^{*}\right\| \nu e^{-\sigma s_{j}}+\left(\epsilon \delta \nu e^{-\sigma s_{j} / 2}\right) / \sigma \\
& +\epsilon(\gamma+\eta) \nu / \sigma+q(p) \nu\left(1-e^{-\sigma s_{j}}\right) / \sigma
\end{aligned}
$$

As $j \rightarrow \infty$, this becomes $\gamma-\eta<r(\gamma+\eta)+q(p) \nu / \sigma$; and since $\eta$ was arbitrary, we get

$$
\gamma \leqslant r \gamma+q(p) \nu / \sigma
$$

from which the result follows.

We remark here that the proof of Theorem 3.2 rests on obtaining a bound on the integral representation of a solution to (3.1) and thus also demonstrates the existence of at least one solution to (3.1).

It is interesting to note at this point that the choice of $D_{r}$, $\xi$ right after inequality (3.4) in the proof could have been less arbitrary. We could have decreased the final bound (3.6) by decreasing $\xi$ and this would have allowed a wider choice of $x_{0}$ to achieve the bound. On the other hand, we could have restricted $x_{0}$ very sharply and allowed a wider latitude with respect to the parameter $p$. Furthermore, the parameter $r$ accelerates this interdependence as it is taken nearer zero. We can paraphrase all this as follows. No matter how small we choose $\|p\|>0$ and $\left\|x_{0}-x^{*}\right\|>0$, we cannot expect to come closer than $\sup _{x \in D}\|G(x)-\bar{G}(x, p)\| \nu / \sigma$ to $x^{*}$. 
4. Numerical Integration of the Continuous Analogue. In the previous section we gave a theorem which established the asymptotic behavior of the continuous analogue. Our purpose here is to first give a certain class of numerical integration procedures which mirror this behavior when applied to the affine part of the problem (3.1). We then show that the effects of nonlinearity and consistent approximations are just the same for these numerical solutions as for the analytic solutions.

We assume that the reader is familiar with linear multistep methods for initial value problems, and we present only the following definition which generalizes one given in [1] for a class of methods having the desired properties.

Definition 4.1. A linear multistep method is weakly A-stable if for any matrix $A$ which satisfies the hypothesis of Lemma 3.1, there exist positive constants $\underline{t}<\bar{t}$ for which the following is true. If the method with step lengths $t_{k} \in[t, \bar{t}], k=0,1$, $\ldots$, is applied to

$$
x^{\prime}(s)=-A\left[x-x^{*}\right], \quad x(0)=x_{0}, \quad 0 \leqslant s, \quad x^{*} \text { arbitrary },
$$

then the sequence $\left\{x_{k}\right\}$ generated by the method converges to $x^{*}$.

We mentioned in Section 1 that all the methods of interest here can be viewed as Euler's method applied to (1.6). Hence we will restrict our attention to Euler's method.

Lemma 4.2. Euler's method is weakly A-stable.

Proof. Let $x^{*}$ be arbitrary and $e_{k}=x_{k}-x^{*}$. Apply Euler's method with step size $t_{k}$ to $x^{\prime}(t)=-A\left[x-x^{*}\right], x(0)=x_{0}$ to obtain

$$
e_{k+1}=e_{k}-t_{k} A e_{k}=\left[\prod_{i=0}^{k}\left(I-t_{i} A\right)\right] e_{0} .
$$

Thus, $\left\{\left\|e_{k}\right\|\right\}$ converges to zero if the spectral radius of $I-t_{i} A$ is uniformly bounded by some number less than 1 . Let $\lambda_{j}=a_{j}+i b_{j}$ be any eigenvalue of $A$. Then $a_{j}>0$ and $\mu_{j}=1-t a_{j}-i t b_{j}$ is the corresponding eigenvalue of $I-t A$ and $\left|\mu_{j}\right|^{2}<1$ for any $t \in\left(0,2 a_{j} /\left|\lambda_{j}\right|^{2}\right)$. Thus, take $0<\underline{t}<\bar{t}=\min _{1 \leqslant i \leqslant n} 2 a_{i} /\left|\lambda_{i}\right|^{2}$ and the proof is complete.

The following theorem is a partial extension of a result in [1]. It shows that even in the more general problem (3.1), the desired results hold for step lengths controlled by the affine term. Roughly, this is because this term dominates as $s$ gets large.

THEOREM 4.3. Assume the conditions of Theorem 3.2 and let $0<\underline{t}<\bar{t}<$ $\min \left\{2 a /|\lambda|^{2}: \lambda=a+i b\right.$ is an eigenvalue of $\left.A\right\}$.

Then there are constants $\widetilde{\xi}, \zeta>0$ and a neighborhood $\widetilde{D}_{r}$ of $x^{*}$ such that any solution to

$$
\begin{aligned}
x_{k+1} & =x_{k}-t_{k} \widetilde{G}\left(x_{k}, p\right), & x_{0} \in \widetilde{D}_{r}, \\
\|p\| & \leqslant \widetilde{\xi}, \quad t_{k} \in[\underline{t}, \bar{t}], & k>0,
\end{aligned}
$$

has the property that

$$
\varlimsup_{k \rightarrow \infty}\left\|x_{k}-x^{*}\right\| \leqslant\left[\sup _{x \in D}\|G(x)-\widetilde{G}(x, p)\|\right] \zeta / \sigma(1-r),
$$

where $\sigma$ is defined for $A$ in Lemma 3.1 .

Proof. Define $e_{k}=x_{k}-x^{*}$ and rewrite (4.3) as 


$$
e_{k+1}-\left(I-t_{k} A\right) e_{k}=t_{k}\left[A e_{k}-G\left(x_{k}\right)+G\left(x_{k}\right)-\widetilde{G}\left(x_{k}, p\right)\right]=t_{k} \cdot d_{k}
$$

which defines $d_{k}$. Now in a manner similar to that in [11], we construct a solution to (4.4) in the form $e_{k}=y_{k}+z_{k}$, where $y_{k}$ is a solution to the homogeneous problem ((4.4) with $\left.d_{k} \equiv 0\right)$ satisfying the initial condition and $z_{k}$ is a particular solution to (4.4) with zero initial data. The reader may verify that $y_{k}$ and $z_{k}$ are given as, follows.

Let $M_{i 0}=I, i \geqslant 0$, and

Then, $y_{k}=M_{k k} e_{0}, k \geqslant 0$, and

$$
M_{i j}=\prod_{m=0}^{j-1}\left(I-t_{i-1-m} A\right), \quad j>0 .
$$

$$
z_{0}=0, \quad z_{k}=\sum_{i=1}^{k} M_{k, k-1} t_{i-1} d_{i-1}, \quad k \geqslant 0 .
$$

For any $\epsilon$ there is a $\delta>0$ such that for $\left\|e_{j}\right\| \leqslant \delta$ we have $\left\|d_{j}\right\| \leqslant \epsilon\left\|e_{j}\right\|+q(p)$, where again $q(p)=\sup _{x \in D}\|G(x)-\widetilde{G}(x, p)\|$. We now have that if $\left\|e_{j}\right\|<\delta, j=0$, ..., $k-1$,

$$
\left\|e_{k}\right\| \leqslant\left\|y_{k}\right\|+\left\|z_{k}\right\| \leqslant\left\|y_{k}\right\|+\bar{t} \sum_{i=1}^{k}\left\|M_{k, k-1}\right\| \cdot\left[\epsilon\left\|e_{i-1}\right\|+q(p)\right] .
$$

Then, since $\left\|y_{k}\right\|$ is monotonically decreasing and $y_{0}=e_{0}$, it follows that

$$
\max _{0 \leqslant i \leqslant k}\left\|e_{i}\right\| \leqslant\left\|e_{0}\right\|+\bar{t} \max _{0 \leqslant i \leqslant k}\left\|e_{i}\right\| \sum_{i=1}^{k}\left\|M_{k, k-i}\right\|+\bar{t} q(p) \sum_{i=1}^{k}\left\|M_{k, k-1}\right\| .
$$

Now $\sum_{i=1}^{k}\left\|M_{k, k-i}\right\|$ can be bounded as follows. Let $t^{*}$ maximize $\|I-t A\|$ for $t \in[t, \bar{t}]$. Then for $\bar{M}=I-t^{*} A,\|\bar{M}\|<1$ by Lemma 4.2 and

$$
\sum_{i=1}^{k}\left\|M_{k, k-1}\right\| \leqslant \sum_{i=1}^{k}\|\bar{M}\|^{k-1}<\sum_{i=0}^{\infty}\|\bar{M}\|^{i}=\frac{1}{1-\|\bar{M}\|} \equiv \zeta .
$$

Thus, from (4.6)

$$
\max _{0 \leqslant i \leqslant k}\left\|e_{i}\right\| \leqslant\left(\left\|e_{0}\right\|+\bar{t} q(p) \zeta\right) /(1-\bar{t} \epsilon \zeta)
$$

and for $\epsilon=r / \overline{t \zeta}$ and $\left\|e_{0}\right\|$ and $q(p)$ sufficiently small, $\max _{0 \leqslant i \leqslant k}\left\|e_{i}\right\|<\delta$ for all $k$.

We now compute $\overline{\lim }_{k \rightarrow \infty}\left\|x_{k}\right\| \equiv \gamma$. For every $\eta>0$ there is an integer $k_{\eta}$ and an infinite set of integers $N=\left\{i: i \geqslant k\right.$ and $\left.\left\|e_{i}\right\|>\gamma-\eta\right\}$. Also, there is an integer $\hat{k}_{\eta}$ such that $\gamma+\eta>\left\|e_{i}\right\|$ for all $i>\hat{k}_{\eta}$. We may rewrite (4.5) as

$$
\left\|e_{i}\right\| \leqslant\left\|y_{i}\right\|+\sum_{j=1}^{i / 2}\left\|M_{i, i-j}\right\| \cdot\left\|d_{j}\right\|+\sum_{j=i / 2+1}^{i}\left\|M_{i, i-j}\right\| \cdot\left\|d_{j}\right\| ;
$$

and thus, for all $i \in N$ such that $i / 2>\hat{k}_{\eta}$,

$$
\gamma-\eta<\left\|e_{i}\right\| \leqslant\left\|y_{i}\right\|+\bar{t} \epsilon \delta \sum_{j=1}^{i / 2}\left\|M_{i, i-j}\right\|+\bar{t} \epsilon(\gamma+\eta) \zeta+\bar{t} q(p) \zeta .
$$

As $i \rightarrow \infty, \Sigma_{j=1}^{i / 2}\left\|M_{i, i-j}\right\| \rightarrow 0$ and $y_{i} \rightarrow 0$. Therefore, in the limit as $i \rightarrow \infty$,

$$
\gamma-\eta \leqslant \bar{t} \epsilon(\gamma+\eta) \zeta+\bar{t} q(p) \zeta \text {. }
$$

But (4.7) must hold for all $\eta$; and therefore, $\gamma \leqslant \bar{t} q(p) \zeta /(1-\overline{t \epsilon} \zeta)$, and the result follows. 
Note that here, as in the case of Theorem 3.2, the proof also implies the existence of at least one solution.

If $A$ is symmetric and $\bar{t}$, instead of being maximized, is chosen so that the eigenvalues of $I-\bar{t} A$ are positive, then $\zeta=1 / \bar{t} \sigma$ where $\sigma$ is the smallest eigenvalue of $A$. Moreover, the result is then identical to Theorem 3.2, since $\nu=1$ in this case.

In some cases, e.g. Newton's method (cf. Section 5.2), the use of consistent approximations does not prevent convergence of the iteration to $x^{*}$. In these cases, the expression $[G(x)-\widetilde{G}(x, p)]$ satisfies a stronger condition which enables it to be handled in the same manner as the term $\left[A\left(x-x^{*}\right)-G(x)\right]$. We state the following useful corollary.

Corollary 4.4. Assume the hypotheses of Theorem 4.3 and let the following condition hold: For every $\epsilon>0$ there exists $\delta>0$ and a vector $p$ such that $\|G(x)-\widetilde{G}(x, p)\| \leqslant \epsilon\left\|x-x^{*}\right\|$ for $\left\|x-x^{*}\right\| \leqslant \delta$. Then, any solution of (4.3) tends to $x^{*}$ as $k \rightarrow \infty$.

5. Application to Specific Methods. In this section, we will identify each of the methods of Section 1 with Euler's linear multistep method in order to apply Theorem 4.3. The results give insight into the effect of using a consistent approximation to one of the well-known methods. Our intent is really more negative than positive; and it is to warn that for some problems, such as unconstrained minimization and nonlinear least squares, the parameter which controls the approximation must be allowed to get small in direct proportion to the accuracy required in the final answer. Our bounds will always include a constant factor $C$, which is a device which allows us to avoid cluttering details about the particular norms used in various parts of the bounds.

\subsection{Steepest Descent.}

THEOREM 5.1. Let $f: D \subset R^{n} \rightarrow R_{1}^{1}$ be Fréchet differentiable on the open convex set $D$. Assume $x^{*} \in D$ has the properties that $\nabla f\left(x^{*}\right)=0$ and $f$ has a second Fréchet derivative $H^{*}$ at $x^{*}$ which is symmetric and positive definite with smallest eigenvalue $\sigma$. Let $r \in(0,1)$ and $\bar{t}$ be a positive number smaller than $2 /\left\|H^{*}\right\|_{2}$. Then there exist positive constants $\rho$ and $\xi$ and a region $D_{r}$ such that any solution of

$$
\begin{gathered}
x_{k+1}=x_{k}-t_{k} \Delta f\left(x_{k}, h\right), \\
x_{0} \in D_{r}, \quad\|h\| \leqslant \xi, \quad t_{k} \in[\underline{t}, \bar{t}], \quad k \geqslant 0,
\end{gathered}
$$

where $0<\underline{t} \leqslant \bar{t}$, satisfies

$$
\varlimsup_{k \rightarrow \infty}\left\|x_{k}-x *\right\| \leqslant \sup _{x \in D}\|\Delta f(x, h)-\nabla f(x)\| \rho / \sigma(1-r) .
$$

Furthermore, if there are constants $K, \alpha>0$, such that for every $x, y \in D, \| \nabla f(x)-$ $\nabla f(y)\|\leqslant K\| x-y \|^{\alpha}$, then

$\varlimsup_{k \rightarrow \infty}\left\|x_{k}-x^{*}\right\| \leqslant C K\|h\|^{\alpha} \rho / \sigma(1-r)$, for some $C>0$ independent of $h$.

Proof. We write (5.1) as

$$
\begin{aligned}
x_{k+1}= & x_{k}-t_{k} H^{*}\left(x_{k}-x^{*}\right)+t_{k}\left[\nabla f\left(x^{*}\right)-\nabla f\left(x_{k}\right)+H^{*}\left(x_{k}-x^{*}\right)\right] \\
& +t_{k}\left[\nabla f\left(x_{k}\right)-\Delta f\left(x_{k}, h\right)\right] .
\end{aligned}
$$


By identifying $H^{*}$ with $A, \nabla f(x)$ with $G(x)$ and $\Delta f(x, h)$ with $\widetilde{G}(x, p)$, the result follows from an application of Theorem 4.3 to problem (5.1).

COROLlaRY 5.2. Let the hypotheses of Theorem 5.1 hold and assume that for some constants $L, \bar{\alpha}$, and every $x, y \in D, f$ is twice differentiable and

$$
\left\|f^{\prime \prime}(x)-f^{\prime \prime}(y)\right\| \leqslant L\|x-y\|^{\bar{\alpha}}, \quad \bar{\alpha} \in(0,1] .
$$

Then, any solution $\left\{x_{k}\right\}$ to the difference equation (5.1) satisfies

$$
\varlimsup_{k \rightarrow \infty}\left\|x_{k}-x^{*}\right\| \leqslant \frac{C \rho\|h\|}{(1-r)}\left[k_{2}\left(H^{*}\right)+\frac{L}{\sigma} \sup _{x \in D}\left\|x-x^{*}\right\|^{\bar{\alpha}}\right] .
$$

Proof. The result follows easily from the well-known fact that a first order Lipschitz constant for a function in a convex region can be taken as a bound on the norm of the derivative of the function in the same region. Thus, in the conclusion of Theorem 5.1 we can take $\alpha=1$ and

$$
\begin{aligned}
K=\sup _{x \in D}\left\|f^{\prime \prime}(x)\right\|_{2} & =\left\|H^{*}\right\|_{2}+\sup _{x \in D}\left\|f^{\prime \prime}(x)-H^{*}\right\|_{2} \\
& \leqslant\left\|H^{*}\right\|_{2}+\sup _{x \in D} L\left\|x-x^{*}\right\|_{2}^{\bar{\alpha}} .
\end{aligned}
$$

Of course, $\left\|H^{*}\right\|_{2} / \sigma=k_{2}\left(H^{*}\right)$.

The main point of this paper lies in expressions like (5.3). If you use a fixed step size finite-difference gradient to try to minimize even a very smooth function $(\bar{\alpha}=1)$, then the distance by which you should expect to miss the minimizing point is directly proportional to the magnitude of the perturbation used in the gradient approximation. Furthermore, the constant of proportionality is composed of two parts; one depends on the conditioning of the quadratic Taylor approximation to $f$ near the minimum, and the other depends jointly on how much $f$ actually deviates from that quadratic model ( $L=0$ if and only if $f$ is quadratic) and how far one starts from the minimizing point.

Now, clearly it is not always impossible to find $x^{*}$ using fixed size finite-difference gradient, but rather the point is that neither is it necessarily always possible. Dennis [6] considered the strictly convex quadratic function $f(x)=x^{T} H^{*} x$ where

$$
H^{*}=\left(\begin{array}{rr}
2.6 & -2.4 \\
-2.4 & 2.5
\end{array}\right) \text {. }
$$

The iteration $x_{k+1}=x_{k}-t_{k} \Delta f\left(x_{k}, 10^{-3} x_{0}\right), x_{0}=(100,105)^{T}$ was carried out in double precision (APL) on Cornell University's IBM 360/65. In this computation, $t_{k}$ was computed from a formula for the exact minimizer for $f\left(x_{k}-t \Delta f\left(x_{k}, 10^{-3} x_{0}\right)\right.$ ). The choice of $h$ or $p=10^{-3} x_{0}$ was made because it is often used in practice.

Let $\theta_{k}$ be the angle between $\Delta f\left(x_{k}, h\right)$ and $\nabla f\left(x_{k}\right)$. Initially, the approximate gradient was excellent with $\cos \theta_{0}=.99998$. After forty-two iterations, the progress towards the minimum at the origin was excellent with $\cos \theta_{42} \doteq .75$ and $x_{42}=$ $(.34678, .38640)^{T}$. After fifty iterations, the method had fallen into the trap predicted by the theory with $x_{50}=(.00083, .00490)^{T}, \cos \theta_{50} \doteq-4 \times 10^{-7}$ and convergence to six decimal places apparent. If we had switched to a central difference at this point, it would probably have been possible to decrease the function a bit further.

5.2. Newton's Method. In this case, it is well known that it is even possible 
to implement the finite-difference analogue in such a way as to preserve the second order convergence of the original method. See [6]. We include this result for completeness and because it allows a greater latitude of step size than previous results.

THEOREM 5.2. Let $F: D \subset R^{n} \rightarrow R^{n}$ have a continuous Fréchet derivative $F^{\prime}(x)$ on the open convex set $D_{0}$. Assume $x^{*} \in D$ is such that $F\left(x^{*}\right)=0$ and that $F^{\prime}\left(x^{*}\right)^{-1}$ exists. Then there exist a positive constant $\xi$ and a region $D_{r}$ such that if $\underline{t} \in(0,2)$ then any solution of

$$
\begin{gathered}
x_{k+1}=x_{k}-t_{k} \Delta F\left(x_{k}, h\right)^{-1} F\left(x_{k}\right), \\
x_{0} \in D_{r}, \quad\|h\| \leqslant \xi, \quad t_{k} \in[\underline{t}, 2), \quad k \geqslant 0,
\end{gathered}
$$

satisfies $x_{k} \rightarrow x^{*}$.

Proof. We write (5.4) as

$$
\begin{aligned}
x_{k+1}-x^{*} & =x_{k}-t_{k}\left(x_{k}-x^{*}\right)-t_{k} F^{\prime}\left(x_{k}\right)^{-1}\left[F\left(x_{k}\right)-F\left(x^{*}\right)-F^{\prime}\left(x_{k}\right)\left(x_{k}-x^{*}\right)\right] \\
& +t_{k}\left[F^{\prime}\left(x_{k}\right)^{-1} F\left(x_{k}\right)-\Delta F\left(x_{k}, h\right)^{-1} F\left(x_{k}\right)\right] .
\end{aligned}
$$

We again identify $I$ with $A, F^{\prime}(x)^{-1} F(x)$ with $G(x)$ and $\Delta F(x, h)^{-1} F(x)$ with $\widetilde{G}(x, p)$. Now, however,

$$
\begin{aligned}
& \left\|F^{\prime}(x)^{-1} F(x)-\Delta F(x, h)^{-1} F(x)\right\| \leqslant\left\|F^{\prime}(x)^{-1}-\Delta F(x, h)^{-1}\right\| \cdot\|F(x)\| \\
& \quad \leqslant\left\|F^{\prime}(x)^{-1}\right\| \cdot\left\|F^{\prime}(x)-\Delta F(x, h)\right\| \cdot\left\|\Delta F(x, h)^{-1}\right\| \cdot\left\|F(x)-F\left(x^{*}\right)\right\| .
\end{aligned}
$$

Since $F^{\prime}\left(x^{*}\right)^{-1}$ exists we may assume without loss of generality that $F(x)^{-1}$. exists and is uniformly bounded on $D$. Similarly, by the consistency assumption, we may as well assume $\Delta F(x, h)^{-1}$ exists and is uniformly bounded on $D$ for $\|h\|$ sufficiently small. The consistency assumption also implies that there is a function $q(h)$ such that $\left\|F^{\prime}(x)-\Delta F(x, h)\right\| \leqslant q(h)$ and $q(h) \rightarrow 0$ as $\|h\| \rightarrow 0$ uniformly for $x \in D$. Thus, there is a constant $n \geqslant 0$ such that

$$
\left\|F^{\prime}(x)^{-1} F(x)-\Delta F(x, h)^{-1} F(x)\right\| \leqslant \eta q(h)\left\|x-x^{*}\right\| .
$$

Now for any $\epsilon>0$ there is an $h$ such that

$$
\left\|F^{\prime}(x)^{-1} F(x)-\Delta F(x, h)^{-1} F(x)\right\| \leqslant \epsilon\left\|x-x^{*}\right\|,
$$

and the result now follows immediately from Corollary 4.4 .

5.3. Levenberg-Marquardt/Gauss-Newton. In Section 2 we proved that the finitedifference Levenberg-Marquardt iteration could be viewed as a consistent approximation to the Ben-Israel iteration which is the same as the Gauss-Newton iteration if $F^{\prime}(x)$ has full column rank. The following theorem gives the appropriate convergence result.

THEOREM 5.3. Let $F: D \subset R^{n} \rightarrow R^{m}, m \geqslant n$, have a continuous Fréchet first derivative at each point $x$ of the open convex set $D$, and let the rank be a constant independent of $x$. In addition, let $x^{*} \in D$ have the properties that it is a zero of $F^{\prime}(x)^{T} F(x)$ and that this function has a positive definite Fréchet derivative at $x^{*}$ with largest eigenvalue $\lambda_{1}$. Let the smallest eigenvalue of $F^{\prime}\left(x^{*}\right)^{T} F^{\prime}\left(x^{*}\right)$ be $\lambda_{2}$.

Then for any $r \in(0,1)$ and any $\mu>0$, there exist constants $\sigma, \xi, \rho$ and a neighborhood $D_{r}$ of $x^{*}$ such that any solution of 


$$
\begin{gathered}
x_{k+1}=x_{k}-t_{k}\left(\mu I+\Delta F\left(x_{k}, h\right)^{T} \Delta F\left(x_{k}, h\right)\right)^{-1} \Delta F\left(x_{k}, h\right)^{T} F\left(x_{k}\right), \\
x_{0} \in D_{r}, \quad\|h\| \leqslant \xi, \quad t_{k} \in[\underline{t}, 2 / \lambda], \quad \underline{t}>0,
\end{gathered}
$$

where $\lambda$ is the largest eigenvalue of

and $2 / \lambda \leqslant 2\left(\mu+\lambda_{2}\right) / \lambda_{1}$ satisfies

$$
\left(\mu I+F^{\prime}\left(x^{*}\right)^{T} F^{\prime}\left(x^{*}\right)\right)^{-1}\left[F^{\prime}\left(x^{*}\right)^{T} F^{\prime}\left(x^{*}\right)+\sum_{i=1}^{m} f_{i}\left(x^{*}\right) f_{i}^{\prime \prime}\left(x^{*}\right)\right]
$$

$$
\varlimsup_{k \rightarrow \infty}\left\|x_{k}-x^{*}\right\| \leqslant\left[\sup _{x \in D_{r}}\left\|F^{\prime}(x)-\Delta F(x, h)\right\|\right] \rho / \sigma(1-r) .
$$

Furthermore, if for some $K, \alpha>0$ and every $x, y \in D,\left\|F^{\prime}(x)-F^{\prime}(y)\right\| \leqslant K\|x-y\|^{\alpha}$, then for some $C>0$, independent of $h$,

$$
\varlimsup_{k \rightarrow \infty}\left\|x_{k}-x^{*}\right\| \leqslant C \cdot K\|h\|^{\alpha} \rho / \sigma(1-r) .
$$

Proof. The proof follows simply from Theorem 4.3 once we identify $G$ with $\left(\mu I+F^{\prime}(x)^{T} F^{\prime}(x)\right)^{-1} F^{\prime}(x)^{T} F(x), A$ with

$$
G^{\prime}\left(x^{*}\right)=\left(\mu I+F^{\prime}\left(x^{*}\right)^{T} F^{\prime}\left(x^{*}\right)\right)^{-1}\left[\left(F^{\prime}\left(x^{*}\right)^{T} F^{\prime}\left(x^{*}\right)+\sum_{i=1}^{m} f_{i}\left(x^{*}\right) f_{i}^{\prime \prime}\left(x^{*}\right)\right],\right.
$$

and $\widetilde{G}(x, p)$ with $\widetilde{L}(x,(\mu, h))$. Note that if the rank of $F^{\prime}(x)$ is not $n$, then $\mu$ may not be taken to be 0 .

In the full rank case, we may take $\mu=0$ and obtain the convergence of the GaussNewton iteration. One interesting sidelight is the following corollary.

COROLlaRY 5.4. Under the hypotheses of Theorem 5.3 with $F^{\prime}(x)$ of rank $n$, if the largest eigenvalue of

$$
\left[F^{\prime}\left(x^{*}\right)^{T} F^{\prime}\left(x^{*}\right)\right]^{-1}\left[F^{\prime}\left(x^{*}\right)^{T} F^{\prime}\left(x^{*}\right)+\sum_{i=1}^{m} f_{i}\left(x^{*}\right) f_{i}^{\prime \prime}\left(x^{*}\right)\right]<2,
$$

then the Gauss-Newton (with $t_{k}=1$ ) iteration is locally convergent to $x^{*}$.

Proof. Take $p=0$ and notice that $\bar{t} \geqslant 1$.

The reader will find a similar but less general condition in [4], namely that $K\left\|F\left(x^{*}\right)\right\|<\lambda_{1}$. The condition is, of course, to be expected since it is the same as the spectral radius of $G^{\prime}\left(x^{*}\right)$ less than 1 ; and so, it would be predicted by Ostrowski's Theorem [18].

We complete this section by giving a convergence proof for the Ben-Israel iteration in the rank deficient case. To do this, however, we need a somewhat stronger condition on $F$. This is necessary to compensate for the fact that at $x *$ the $A$ matrix $\left(G^{\prime}\left(x^{*}\right)\right.$ for $\left.G(x)=F^{\prime}(x)^{+} F(x)\right)$ is singular, which means that the asymptotic character of the solution is determined by the perturbations. An alternate approach, to handle this and other singularities, is given in [2].

THEOREM 5.5. Assume the conditions of Theorem 5.3 except that the rank of $F^{\prime}(x)$ may be less than $n$. Assume the conditions of Corollary 2.4 with $\beta>1$. Then for any $r \in(0,1)$ and for any $\mu>0$, there is a neighborhood $D_{r}$ such that any solution of

$$
\begin{gathered}
x_{k+1}=x_{k}-t_{k} F^{\prime}\left(x_{k}\right)^{+} F\left(x_{k}\right), \\
x_{0} \in D_{r}, \quad t_{k} \in\left[\underline{t}, 2 \mu / \lambda_{i}\right], \quad \underline{t}>0, \lambda \text {, as in Theorem } 5.3
\end{gathered}
$$

tends to $x^{*}$ as $k \rightarrow \infty$. 
Proof. Identify $\widetilde{G}(x, \mu)$ with $\left(\mu I+F^{\prime}(x)^{T} F^{\prime}(x)\right)^{-1} F^{\prime}(x)^{T} F(x), A$ with $G_{x}\left(x^{*}, \mu\right)$ and $G(x)$ with $F^{\prime}(x)^{+} F^{\prime}(x)$. Then apply Corollaries 2.4 and 4.4.

Note that to use an arbitrarily large step length we must, by Corollaries 2.4 and 4.4 , start correspondingly close to $x^{*}$.

U. S. Army Research Office

P. O. Box 12211

Research Triangle Park, North Carolina 27709

Department of Computer Science

Cornell University

Ithaca, New York 14853

1. P. T. BOGGS (1971) "The solution of nonlinear operator equations by $A$-stable integration techniques," SIAM J. Numer. Anal., v. 8, pp. 767-785. MR 45 \#6179.

2. P. T. BOGGS (1976) "The convergence of the Ben-Israel iteration for nonlinear least squares problems," Math. Comp. (To appear.)

3. W. E. BOSARGE, JR. (1968) Infinite Dimensional Iterative Methods and Applications, IBM Publications 230-2347, Houston.

4. K. M. BROWN \& J. E. DENNIS, JR. (1970), "Derivative-free analogues of the LevenbergMarquardt and Gauss algorithms for nonlinear least squares approximation," Numer. Math., v. 18, pp. 289-297. MR 46 \#2859.

5. E. A. CODDINGTON \& N. LEVINSON (1955) Theory of Ordinary Differential Equations, McGraw-Hill, New York. MR 16, 1022.

6. J. E. DENNIS, JR. (1971) "Algorithms for nonlinear problems which use discrete approximations to derivatives," Proc. ACM 1971 Nat'l. Conference, Chicago.

7. J. E. DENNIS, JR. (1970) "On the convergence of Newton-like methods," Numerical Methods for Nonlinear Algebraic Equations, edited by Philip Rabinowitz, Gordon-Breach, New York.

8. M. K. GAVURIN (1968) "Nonlinear functional equations and continuous analogs," Izv. Vyšs. Učebn. Zaved. Matematika, v. 1958, no. 5 (6), pp. 18-31; English transl., Technical Report 68-70, University of Maryland, College Park, Md. MR 25 \#1380.

9. A. A. GOLDSTEIN (1967), Constructive Real Analysis, Harper \& Row, New York. MR 36 \#705.

10. G. H. GOLUB \& V. PEREYRA (1972), "The differentiation of pseudo inverses and nonlinear least squares problems whose variables separate," SIAM J. Numer. Anal., v. 10, pp. 413-432. MR 49 \#1753.

11. P. HENRICI (1962), Discrete Variable Methods in Ordinary Differential Equations, John Wiley, New York. MR 24 \#B1772.

12. J. HURT (1967) "Some stability theorems for ordinary difference equations," SIAM J. Numer. Anal., v. 4, pp. 582-596. MR 36 \#4839.

13. K. LEVENBERG (1944) "A method for the solution of certain non-linear problems in least squares," Quart. Appl. Math., v. 2, pp. 164-168. MR 6, 52.

14. D. W. MARQUARDT (1963) "An algorithm for least-squares estimation of nonlinear parameters," J. Soc. Indust. Appl. Math., v. 11, pp. 431-441. MR 27 \#3040.

15. GUNTER H. MEYER (1968), "On solving nonlinear equations with a one-parameter operator imbedding," SIAM J. Numer Anal., v. 5, pp. 739-752. MR 39 \#3697.

16. J. M. ORTEGA (1973) "Stability of difference equations and convergence of iterative processes," SIAM J. Numer. Anal., v. 10, pp. 268-282. MR 49 \#4281.

17. J. M. ORTEGA \& W. C. RHEINBOLDT (1970) Iterative Solution of Nonlinear Equations in Several Variables, Academic Press, New York. MR 42 \#8686.

18. A. M. OSTROWSKI (1966) Solution of Equations and Systems of Equations, 2nd ed., Pure and Appl. Math., vol. 9, Academic Press, New York. MR 35 \#7575.

19. C. RADHAKRISHNA RAO \& SUJIT KUMAR MITRA (1971) Generalized Inverse of Matrices and Its Applications, John Wiley, New York. MR 49 \#2780.

20. R. A. TAPIA (1971) "The differentiation and integration of nonlinear operators," Nonlinear Functional Analysis and Applications (Proc. Advanced Sem., Math. Res. Center, Univ. of Wisconsin, Madison, Wis., 1970), Academic Press, New York, pp. 45-101. MR 44 \#3160. 